\title{
Passage of Single Hydrocarbon Chains through a defect of Carbon Nanotube
}

\author{
M. Koshino, ${ }^{*}$ N. Solin, ${ }^{* *}$ Y. Niimi, ${ }^{*}$ T. Tanaka, ${ }^{* *}$ H. Isobe, ${ }^{* *}$ and E. Nakamura ${ }^{* * *}$ \\ ${ }_{* *}^{*}$ Nakamura Functional Carbon Cluster Project, ERATO, JST, Kawaguchi, Saitama 332-0012, Japan. \\ ** Department of Chemistry, The University of Tokyo, Hongo, Bunkyo-ku, Tokyo 113-0033, Japan.
}

We recently demonstrated that time-resolved, near-atomic resolution imaging by transmission electron microscope (TEM) is a powerful method to study the structural changes of single organic molecules in motion ${ }^{1,2}$. Here, we report that the TEM can also provide with motional images in what structure and orientation hydrocarbon chains pass through a nanosized pore, and how they interact with the pore ${ }^{3}$.

A High-resolution TEM (HRTEM) observation at $293 \mathrm{~K}$ was carried out by JEM-2100F at 120 $\mathrm{kV}$. A series of TEM images was obtained at time intervals of $2.1 \mathrm{~s}$ with an exposure time of $0.5 \mathrm{~s}$ $\left(4.0 \times 10^{4} \mathrm{e} \bullet \mathrm{nm}^{-2}\right)$, followed by a readout time of $1.6 \mathrm{~s}$. An HRTEM observation at $4 \mathrm{~K}$ was carried out by JEM-2100FC with an aberration corrector for the objective lens (CEOS) operated at $120 \mathrm{kV}$. A series of TEM images was obtained at intervals of $1.0 \mathrm{~s}$ using an exposure time of $0.5 \mathrm{~s}\left(1.5 \times 10^{5}\right.$ $\mathrm{e} \bullet \mathrm{nm}^{-2}$ ), followed by a readout time of $0.5 \mathrm{~s}$.

In this study, we synthesized fullerene molecules $\mathbf{1}$ and $\mathbf{2}$ that bear alkyl and alkenyl chain as shown in Fig.1A. Each molecule was encapsulated in a perforated single-wall carbon nanotube (CNT). We performed experiments at 293 and obtained the images of a hydrocarbon chain egress from the tube through the pores into the vacuum space outside the CNT. (Fig.1B, left). We construct model structures of molecules (middle) from the experimental TEM images and, based on the models, simulated TEM images (right). The energy of each modeled structure has small differences (Fig.C). At the stage of $4 \mathrm{~K}$, a similar phenomenon was also observed where a hydrocarbon chain comes out of the tube wall (Fig.1D). We have obtained several important findings. For example, it is found that the observed molecular motions are very slow, with a time scale of seconds. The slow motions of molecules indicate that the molecules are under constraint even at $293 \mathrm{~K}$. As the observed motions are qualitatively at the same time scale between the stage at $293 \mathrm{~K}$ and $4 \mathrm{~K}$, the major energy source of the observed molecular motions would be the electron beam used for the imaging. Our observations can provide molecular-level information on the transport of molecules through nanopores with direct information of the molecular structures. This has not been so far available using any other experimental method. The exploration of chemical interactions between molecules and membrane pores is the subject for future study, in which we can design functional membranes that penetrate particular types of molecules.

Reference

[1] Koshino M. et al. Imaging of single organic molecules in motion. Science 316, 853 (2007).

[2] Solin N. et al. Imaging of aromatic amide molecules in motion. Chem. Lett. 36, 1208-1209 (2007).

[3] M. Koshino et al. Imaging the passage of a single hydrocarbon chain through a nanopore. Nature Nanotech. 3, 595-597 (2008). 
A

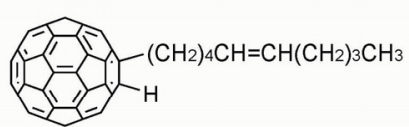

1 (trans isomer)

B
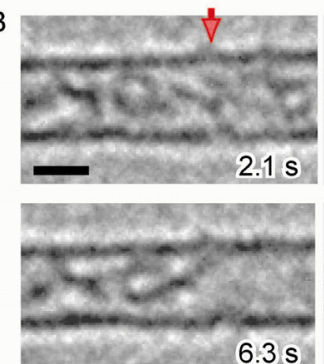

$6.3 \mathrm{~s}$

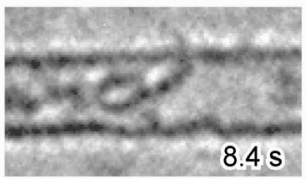

$8.4 \mathrm{~s}$

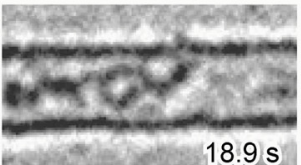

D

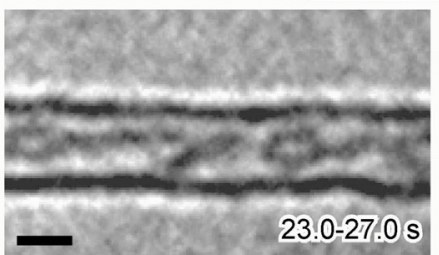

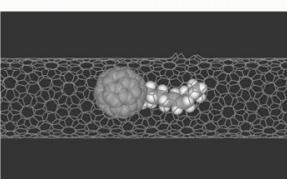
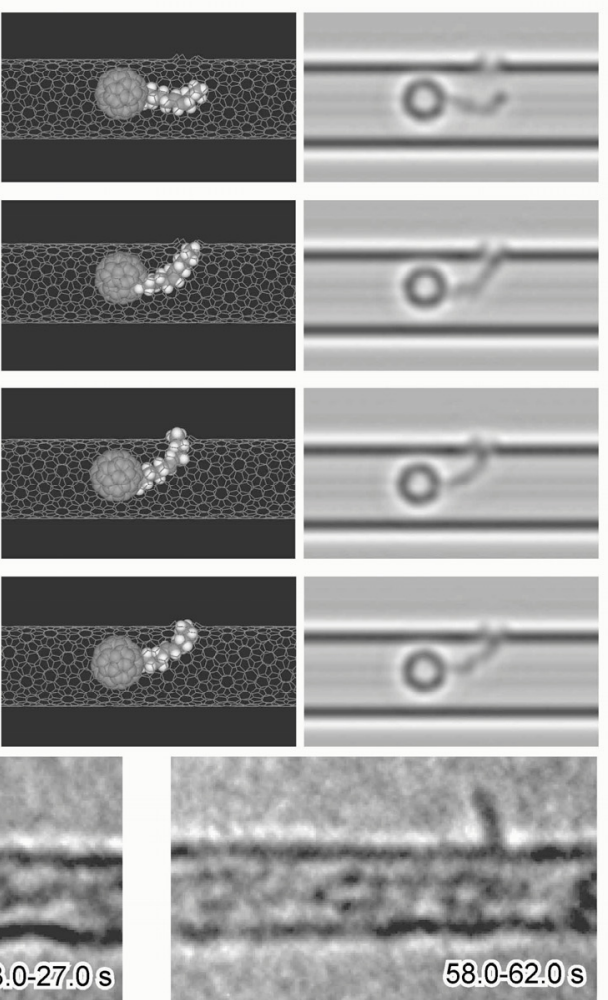
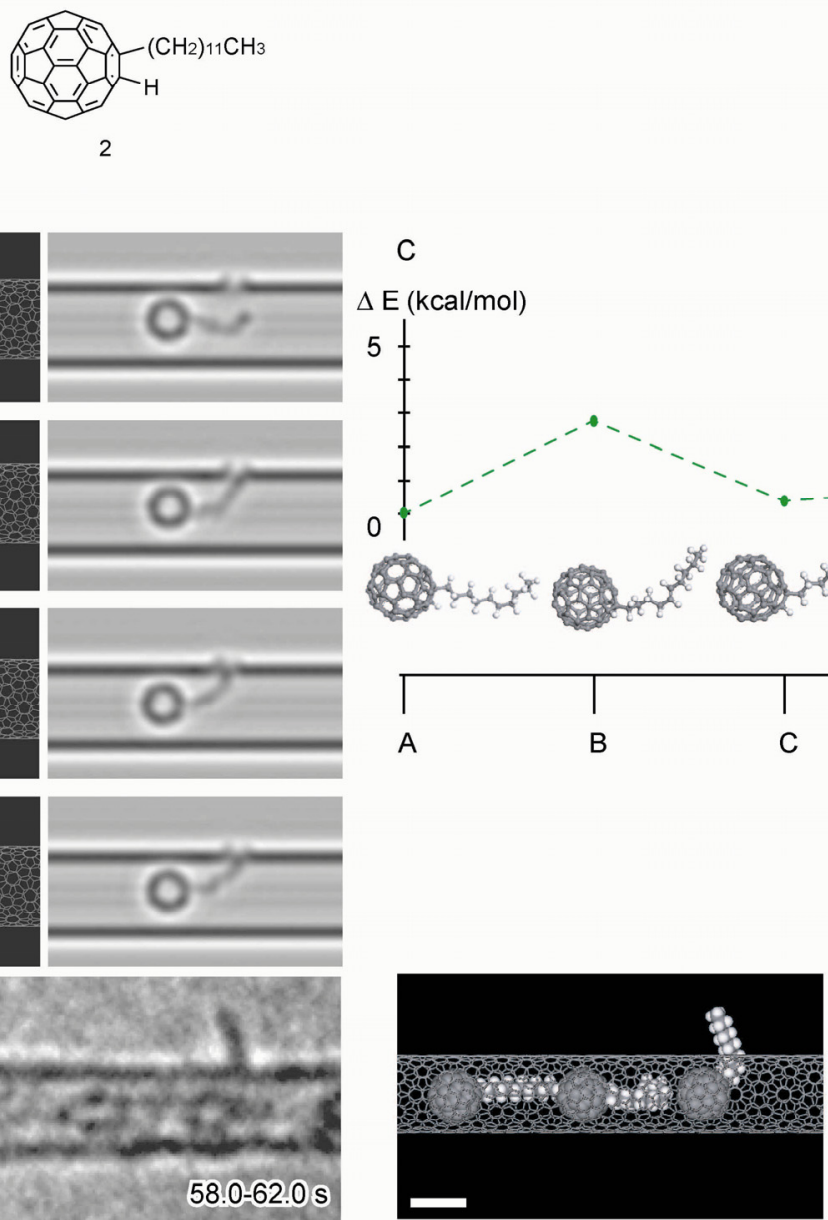

Fig. 1. (A) The sample molecules. $\mathbf{1}=$ alkenyl fullerene, and $\mathbf{2}=$ alkyl fullerene. (B) Alkenyl fullerene 1 sticking out of a $0.5 \mathrm{~nm}$ size pore from a CNT with $1.5 \mathrm{~nm}$ diameter. Models and simulations are shown on the right-hand side. (C) Energy diagram of model structures shows small energy differences during the conformational changes. (D) Alkyl fullerene 2 on a sample stage at $4 \mathrm{~K}$ in which a hydrocarbon chain also comes out of a CNT after $57.0 \mathrm{~s}$ observation. The scale bars represent a distance of $1 \mathrm{~nm}$.

Acknowledgement

This work is performed in collaboration with Nanotube Research Center, National Institute of Advanced Industrial Science and Technology (AIST), Japan. 